\title{
Switched systems as models for dynamic clinical trials
}

\author{
Y. Du AND C. MARTin \\ Dedicated to Wing Wong on the occasion of his 60th birthday
}

\begin{abstract}
The goal of the paper is to make a case for the use of switched systems as a model for the simulation of dynamic clinical trials. Dynamic clinical trials are characterized by the use of different treatments based on the response of a patient to the current treatment. We show that switched systems are, in fact, a suitable model for these trials.
\end{abstract}

Keywords And Phrases: dynamic clinical trials, switching systems, stability.

\section{Introduction}

The choice of a treatment level and its type is one of the most important, yet difficult decisions made by a researcher in a clinical study. Dynamic treatment regimes that provide treatment to patients only if and when the treatment is needed and adjust the level of treatment to the patient's need become the most relevant for this purpose. These regimes are based on decision rules that input patient outcomes collected during treatment and output recommended changes in assignment of the type and level of treatment. To execute this correctly a researcher needs to consistently monitor a patient's reaction to the treatments and adjust the treatment level and type accordingly.

The idea behind dynamic clinical trials is based on the standard treatments of difficult diseases. Drug A is given to the patient and if the patient responds to the treatment then no further action is needed. However if the patient fails to respond in totality to drug A then the treatment will be switched to Drug B. In contrast to classical trials in which every subject is assigned to a fixed treatment, dynamic trials are designed to allow the treatment to be switched between types on the basis of patient response. 
Decision rules for how dosage level and type should vary with time are specified prior to the beginning the trial. The set of decision rules comprises the treatment regime, [16].

There can be many rationales for switching between drug regimes. The patient may not be responding optimally to a treatment and therefore the treatment is changed in order to gain an improved outcome. In HIV treatment, along with many other treatment regimes, there are toxic side effects associated with the long term treatment, [1, 20], and the treatment is switched in order to minimize side effects.

Dynamic treatment regimes are currently being used in designed clinical trials. One example is the Elderly Program randomized trial [3, 5]. In this trial patients were assigned to a dynamic treatment regime that had a goal of minimizing the dosage of treatment needed to maintain a patient's systolic blood pressure (SBP) at or below a predetermined level. At the beginning of the trial all patients were given equal dosages of antihypertensive medication. After 8 weeks, patients whose SBP was higher than the predetermined level had their dosage doubled. After 16 weeks of trial, patients whose SBP was still higher than the predetermined level had a second antihypertensive medication added. After 24 weeks, patients whose SBP continued to exceed the predetermined level had their dose of the second antihypertensive medication doubled. Here the switches are made to change the dosage level or to add a new drug.

A dynamic treatment regime was also employed in the Fast Track Prevention Program, [2, 15]. The Fast Track Prevention Program was a randomized trial of a complex preventive intervention wing versus a control wing. The intervention was designed both to prevent the occurrence of drug use and behavior disorders in children and reduce the level of drug use and behavior disorders if they already existed in children. A dynamic treatment regime was used as a part of this intervention program designed to improve family functioning. This regime was implemented as follows. At the end of each semester, the family counsellor filled out a home visiting process measure (HVPM) consisting of six statements, which described the quality of family functioning. Home visiting assignments for the next semester were determined based on the total score of the HVPM. If the total score of the HVPM was less than or equal to 8 , then the family received 16 home visits during the following semester. If the total score was in between 9 and 16, then the the family received 8 home visits the next semester. A total score of 17 or higher resulted in an assignment of 4 home visits during the following semester. Here the switching is based on changing the level of interaction. 
In [21] a dynamic treatment regime for a trial involving advanced prostrate cancer is analyzed. In that trial there are four treatments and each subject is randomly assigned to a treatment. Based on the response of the subject to the assigned treatment one of the remaining three treatments was randomly assigned. The analysis in that paper is exceptionally complete. It sets a standard for the statistical analysis of such trials.

As we can see from these given examples, dynamic treatment regimes are being successfully used in practice. This paper was motivated by a series of papers by S. A. Murphy, [16 18, on dynamic treatment regimes and is based on the theory of switched linear systems that has received considerable attention in the systems theory literature. Considering the knowledge of both theories the main idea of dynamic treatments through the switched linear systems can be seen as the process of switching between two or more treatments types in order to improve the effect of such a treatment regime as compared with the effect of any single treatment. In this paper we are interested in showing that the theory of switching systems is a natural model for dynamic clinical trials.

\section{Models}

We assume that when a drug is administered to a subject, the subject's response is measured over time. The response can be modelled as the output of a differential equation of the form

$$
\dot{x}(t)=f(x(t), \lambda), \quad y(t)=h(x(t)),
$$

where $y$ is the response and $x$ is the underlying state of the subject. Alternatively the response, if discrete in nature, may be modelled as

$$
x_{n+1}=g\left(x_{n}, \lambda\right), \quad y_{n}=h\left(x_{n}\right)
$$

In both cases the vector $\lambda$ is a vector of parameters for the particular subject. In a simple two drug regime, a suitable model is given by

$$
\dot{x}=\delta(t) f_{1}\left(x(t), \lambda_{1}\right)+(1-\delta(t)) f_{2}\left(x(t), \lambda_{2}\right)
$$

or

$$
x_{n+1}=\delta_{n} g_{1}\left(x_{n}(t), \lambda_{1}\right)+\left(1-\delta_{n}\right) g_{2}\left(x_{n}(t), \lambda_{2}\right) .
$$

There is an extensive literature on such nonlinear systems. See for example the papers and the references therein, [8, 13, 14. 
There are two distinct approaches to these systems in the systems literature. One approach is to treat the switching sequence, or in the case of the stochastic models, the rate of the jump process as controls and select the controls to optimize some criteria. The other approach is to consider them as coming from some class and studying the properties of the system in terms of the class as is done in [8. In this paper we concentrate on the latter and determine various statistical properties of the system. A major difference between the systems theory development and what is needed for clinical trials is that in the systems theory we are interested in the long term behavior of the systems. In clinical trials we almost never have the privilege of more than a very small number of switches and the theory must be developed as to what can be done in a finite horizon.

In this section we provide descriptions of two possible models for dynamic treatment regimes. For purposes of clarity and with some thought towards application we will concentrate on systems that are formed from linear subsystems. The state of the system represents some physiological aspect of interest in the trial. It is usual to impose two bounds on some measure of the state and if either bound is violated the experiment ends, i.e. the bounds form absorbing boundaries for the system. In systems theory we are most often interested in the stability of the system. That is, we want the state of the system to approach zero in some sufficiently fast manner. For applications in clinical trials the situation may, in fact, be different. We may consider the state of zero to represent death or failure and the upper bound to represent the absence of disease or success. In either case the stability analysis is critical.

We consider three basic systems that cover many of the dynamic treatment schedules and physiology where switching occurs.

\subsection{Continuous time with Poisson switching}

In this section we consider the stochastic version of the switched linear system

$$
\dot{x}=(\delta(t) A+(1-\delta(t)) B) x
$$

where

$$
\delta(t) \in\{0,1\}
$$

for all $t$. This deterministic system was studied by Dayawansa and Martin, [8], and they proved that the system is stable for all switching sequences 
$\delta(t)$ if and only if the two systems

$$
\dot{x}=A x \quad \text { and } \quad \dot{x}=B x
$$

have a common Lyapunov function.

We focus on switching sequences generated by a Poisson process. The control for this system is the rate of switching. The resulting system is given by

$$
\begin{aligned}
& d x=((z(t) A+(1-z(t)) B) d x+C d w \\
& d z=(1-2 z(t)) d N_{\lambda}
\end{aligned}
$$

where $N_{\lambda}$ is a Poisson process of rate $\lambda$ and $d w$ is a standard Brownian motion. The Brownian motion process is added for robustness of the modelling process. Note that the process $d z$ takes on only the value of 0 or 1 .

We have the following theorem from [10] which is based on [4].

Theorem 2.1. Consider the system

$$
\begin{aligned}
& d x=(z A+(1-z) B) x d t \\
& d z=(1-2 z) d N
\end{aligned}
$$

where $N$ is a Poisson process with rate $\lambda$. The expected value of $x, E x(t)$ goes to zero for all $\lambda$ if and only if the matrix

$$
\left(\begin{array}{cc}
B-\lambda I & \lambda I \\
\lambda I & A-\lambda I
\end{array}\right)
$$

has all of its eigenvalues in the open left half plane.

Again this is an asymptotic result. This model would represent the uncoordinated switching between treatment types.

\subsection{Discrete time with random switching}

Here we consider the system

$$
x_{n+1}=\left(u_{1} A_{1}+\cdots+u_{k} A_{k}\right) x_{n}
$$

where the $u_{i}$ 's are random variables with $u_{i} \in\{0,1\}, \sum_{i=1}^{k} u_{i}=1$ and $P\left(u_{i}=\right.$ $1)=\frac{1}{k}$ and each $A_{i} \in G L(n, R)$. We note that it is not necessary for the 
probabilities to be uniformly distributed as $\frac{1}{k}$ but it simplifies the notation and the uniform distribution is the most commonly used distribution. This system has been extensively studied both as a switching system and as a model for simple dynamic clinical trials. See the papers, [9, 11, 19, 22, 23] and the references therein.

2.2.1. Stability of system. In this section we will state the following theorem. The proof is found in [11. This theorem provides an easily checkable criterion for almost sure convergence. Other results along these same lines can be found in [6, 7].

Theorem 2.2. If the system

$$
x_{m+1}=\left(\delta_{1, m} A_{1}+\delta_{2, m} A_{2}+\cdots+\delta_{k, m} A_{k}\right) x_{m}
$$

is stable for all choices of the $\delta_{i, m} \in\{0,1\}$ and $\sum_{i} \delta_{i, m}=1$, then

$$
\begin{aligned}
E_{m+1} & =\left(\frac{1}{k} \sum_{i=1}^{k} A_{i}\right) E_{m} \\
C_{m+1} & =\frac{1}{k} \sum_{i=1}^{k} A_{i} C_{m} A_{i}^{\prime}
\end{aligned}
$$

are both stable.

\section{Simulation}

In this section we consider a continuous time system. We assume that this represents the underlying physiology of the subject when subjected to treatments $\mathrm{A}$ and $\mathrm{B}$. In practice we would actually be sampling this system to obtain data that would be used to determine when to switch treatments. We consider two stable systems and our goal is to drive them away from the origin for a few time points. This would be a fair representation of what might be seen in the treatment of a stage 4 oncology patient. That is, we would see initial improvement but would be unable to control the disease for a long time period. However there are diseases in which long term survival is possible by a series of treatment adjustments. Type II Diabetes has long term survival and almost always involves a series of treatment adjustments. 
We will examine the behavior of the system

$$
\frac{d}{d t} x(t)=[\delta(t) A+(1-\delta(t)) B] x(t)
$$

where

$$
\begin{aligned}
A & =\left[\begin{array}{cc}
e^{-0.1 t} \cos (t) & 0.5 e^{-0.1 t} \sin (t) \\
-2 e^{-0.1 t} \sin (t) & e^{-0.1 t} \cos (t)
\end{array}\right] \\
B & =\left[\begin{array}{cc}
e^{-0.1 t} \cos (t) & 2 e^{-0.1 t} \sin (t) \\
-0.5 e^{-0.1 t} \sin (t) & e^{-0.1 t} \cos (t)
\end{array}\right] .
\end{aligned}
$$

These systems are both stable and have the same spectra. Our goal is to keep it as far from 0 as possible for as long as possible by switching between the two systems $\dot{x}=A x$ and $\dot{x}=B x$. We are going to consider these two separate systems.

\subsection{System I}

With $\delta_{n}=0$, the combined system $\frac{d}{d t} x(t)=[\delta(t) A+(1-\delta(t)) B] x(t)$ will change to the system $\frac{d}{d t} x(t)=B x(t)$. We assume the initial condition $x_{0}=$ $[1,0]^{\prime}$. Also, we are going to graph this system in order to describe the behavior of System I.

Then, for the System I

$$
\frac{d}{d t} x(t)=B x(t)
$$

where

$$
B=\left[\begin{array}{cc}
e^{-0.1 t} \cos (t) & 2 e^{-0.1 t} \sin (t) \\
-.5 e^{-0.1 t} \sin (t) & e^{-0.1 t} \cos (t)
\end{array}\right]
$$

The graph is as in Figure 1 and is represented by the dotted line.

Based on the above we find that it starts at the point $(1,0)$, and will converge to the origin as $e^{-.1 t}$. Therefore, the convergence is relatively fast.

\subsection{System II}

Next, we will talk about the second system. For System II, we let $\delta(t)=1$. Then the system will be

$$
\frac{d}{d t} x(t)=A x(t)
$$




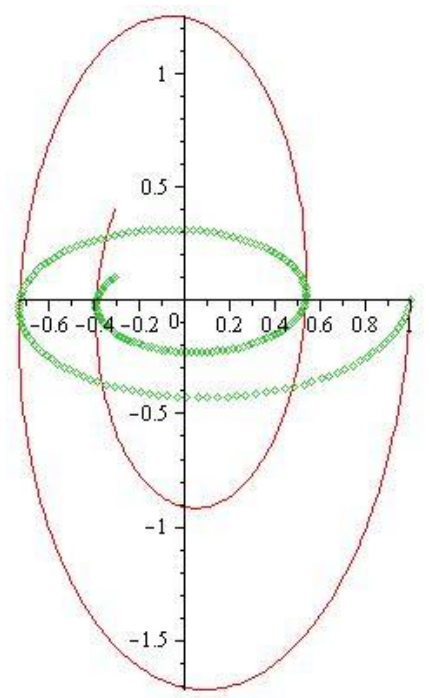

Figure 1: The trajectories of the two systems

where

$$
A=\left[\begin{array}{cc}
e^{-0.1} \cos (t) & .5 e^{-0.1} \sin (t) \\
-2 e^{-0.1} \sin (t) & e^{-0.1} \cos (t)
\end{array}\right]
$$

In the graph of this system in Figure 1 the initial data is the same as for the previous system. This graph is represented by the solid line.

From the graph, we find that even though the plot will converge to the origin at the same rate as before, $e^{-.1 t}$. From the graph we can calculate the switching point that will be used for the combined system.

\subsection{Combined system}

According to the previous two systems, we would like to combine two systems together in order to obtain a model that could be as far from 0 as possible for a short time. Since both systems are stable and there is no common Lyapunov function it is possible to drive the system to infinity. We do not do that here but choose one switching point to get a short term excursion from the origin.

Then we will introduce the strategy to combine the two systems. First of all, we will switch to the System II. We choose to make the switch at the point where the first system crosses the $\mathrm{x}$-axis. We see that the distance from 
the origin decreases for a while and then increases until it crosses the axis and then begins decreasing trajectory. This is not atypical of what would be seen in an oncology patient. We switch at this point and there is an immediate increase in distance from the origin and then when it crosses the y-axis the combined system begins a steady decline to the origin. So this trajectory represents one switch. With a terminal oncology patient there might be one or possibly two more such switches. In this example the switch occurs at the time $t=\pi$.

The graph is as in Figure 2.

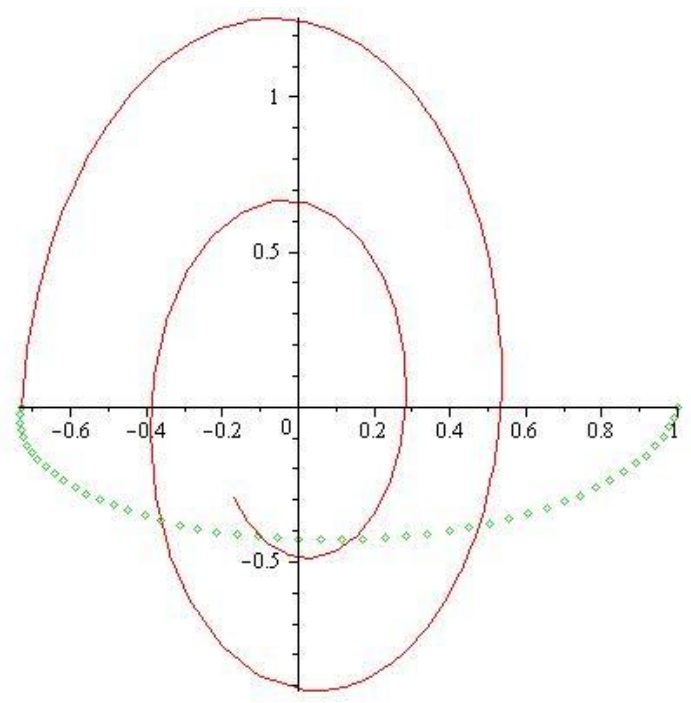

Figure 2: The trajectory of the switched system

It would be more realistic to replace the systems with stochastic differential equations of the form

$$
d x=((\delta(t) A+(1-\delta(t)) B) x(t)) d t+C d W(t)
$$

where $d W(t)$ is Brownian motion. However the goal of the paper is not to make a complete model but to show that the switched system is a good candidate for a model. 


\section{Conclusion}

In this paper we have described three versions of the switching system methodology both in theory and in simulation. We have modelled a simplified version of a trial to show the utility of the modelling techniques. While we have used linear models as the basis for this paper the same approach could be used for nonlinear models of physiological processes. For the purposes of simulation we however prefer the linear models due to their inherent simplicity and their adaptability. There is a great deal known about fitting linear models to data. A much more detailed model is being developed in, [12, based on the PhD dissertation of S. Li.

\section{References}

[1] B. M. Adams, H. T. Banks, M. Davidian and E. S. Rosenberg, Model fitting and prediction with HIV treatment interruption data. Bulletin of Mathematical Biology, 69 (2007), 563-584.

[2] K. L. Bierman and M. T. Greenberg and Conduct Problems Prevention Research Group, Social Skill Training in the Fast Track Program. In: Preventing Childhood Disorders, Substance Abuse, and Delinquency (R. DeV. Peters and R. J. McMahon eds.), Newbury Park, CA: Sage, 1996.

[3] N. O. Borhani, W. B. Applegate, J. A. Cutler, B. R. Davis, C. D. Furberg, E. Lakatos, L. Page, N. M. Perry, W. M. Smith and J. L. Probstfield, Part 1: Rationale and Design. Hypertension, 17 (1991), II2-15.

[4] R. Brockett, Stochastic control. Lecture notes, 2009.

[5] Cooperative Research Group, Rationale and Design of a Randomized Clinical Trial on Prevention of Stroke in Isolated Systolic Hypertension. Journal of Clinical Epidemiology, 41 (1988), 1197-1208.

[6] O. Costa, M. Fragoso and R. Marquez, Discrete-time Markov jump linear systems. London: Springer, 2005.

[7] X. Dai, Y. Huang and M. Xiao, Almost sure stability of discrete-time switched linear systems: a topological point of view. SIAM J. Control Optim., 47 (2008), 2137-2156.

[8] W. P. Dayawansa and C. Martin, A converse Lyapunov theorem for a class of dynamical systems which undergo switching. IEEE Trans. Automat. Control, 44 (1999), no. 4, 751-760. 
[9] Y. Du, Simulation of the optimal timing of a multiple drug regime. Master thesis, Texas Tech University, 2009.

[10] B. Hanlon, V. Tyuryaev and C. Martin, Stability of switched linear systems with Poisson switching. To appear, CIS.

[11] B. Hanlon, N. Wang, M. Egerstedt and C. Martin, Switched linear systems: Stability and the convergence of random products. To appear, CIS.

[12] S. Li and C. Martin, A switching system model of a dynamic treatment regime. In preparation.

[13] D. Liberzon, Switching in systems and control. Systems \& Control: Foundations \& Applications. Birkhäuser Boston, Inc., Boston, MA., 2003.

[14] C. Lobry, Contrôllabilité des systèmes nonlinéaires. SIAM J. Contr. Opt., 8 (1970), 573-605.

[15] R. J. McMahon, N. Slough and Conduct Problems Prevention Research Group, Family-Based Intervention in the Fast Track Program. In: Preventing Childhood Disorders, Substance Abuse, and Delinquency (R. DeV. Peters and R. J. McMahon eds.), Newbury Park, CA: Sage, 1996.

[16] S. A. Murphy, Optimal dynamic Treatment Regimes. Journal of the Royal Statistical Society: Series B (Statistical Methodology), 65 (2003), 331-355.

[17] S. A. Murphy and D. Bingham, Screeing experiments for Developing Dynamic Treatment Regimes. Journal of the ASA, 104 (2009), 391-408.

[18] S. A. Murphy, M. J. van der Laan and J. M. Robins, Marginal mean models for dynamic regimes. J. Amer. Statist. Assoc., 96 (2001), no. 456, 1410-1423.

[19] M. Ptukhina, A comparison of two simulation models in clinical trials. Master thesis, Texas Tech University, 2010.

[20] E. S. Rosenberg, M. Davidian, H. T. Banks, Using mathematical modeling and control to develop structured treatment interruption strategies for HIV infection. Drug and Alcohol Dependence, 88 (2007), S41-S51, Supplement issue on "Customizing Treatment to the Patient: Adaptive Treatment Strategies".

[21] L. Wang, A. Rotnitzky, X. Lin, R. Millikan and P. Thall, Evaluation of viable dynamic treatment regimes in a sequentially randomized trial of advanced prostrate cancer. Preprint. 
[22] N. Wang, Switched systems as models of dynamic treatment scheduling. Master thesis, Texas Tech University, 2009.

[23] N. Wang, M. Egerstedt and C. Martin, Stability of switched linear systems and the convergence of random products. Proceedings of the 48th CDC, 2009.

[24] M. A. Wicks, P. Peleties and R. DeCarlo, Construction of piecewise Lyapunov functions for stabilizing switched systems. In: Proc. 33rd IEEE Conf., 1994.

Division of Biostatistics

University of Texas School of Public Health-Houston

Houston, TX 77030, USA

E-mail address: yining.du@yahoo.com

Department of Mathematics and Statistics

Texas Tech University

LuBBock, TX 79409, USA

E-mail address: clyde.f.martin@ttu.edu

Received February 25, 2012

ACCEPTED June 14, 2012 